\title{
Study of Gamma Ray Exposure Buildup Factor for Some Ceramics with Photon Energy, Penetration Depth and Chemical Composition
}

\author{
Tejbir Singh, ${ }^{1}$ Gurpreet Kaur, ${ }^{2}$ and Parjit S. Singh ${ }^{3}$ \\ ${ }^{1}$ Department of Physics, Sri Guru Granth Sahib World University, Fatehgarh Sahib, Punjab 140407, India \\ ${ }^{2}$ Department of Physics, Maharishi Markandeshwar University, Mullana, Haryana 133207, India \\ ${ }^{3}$ Department of Physics, Punjabi University, Patiala, Punjab 147002, India
}

Correspondence should be addressed to Tejbir Singh; dr.tejbir@gmail.com

Received 12 June 2012; Accepted 7 January 2013

Academic Editor: Baolin Wang

Copyright @ 2013 Tejbir Singh et al. This is an open access article distributed under the Creative Commons Attribution License, which permits unrestricted use, distribution, and reproduction in any medium, provided the original work is properly cited.

\begin{abstract}
Gamma ray exposure buildup factor for some ceramics such as boron nitride (BN), magnesium diboride $\left(\mathrm{MgB}_{2}\right)$, silicon carbide $(\mathrm{SiC})$, titanium carbide $(\mathrm{TiC})$ and ferrite $\left(\mathrm{Fe}_{3} \mathrm{O}_{4}\right)$ has been computed using five parametric geometric progression (G.P.) fitting method in the energy range of 0.015 to $15.0 \mathrm{MeV}$, up to the penetration of 40 mean free path ( $\mathrm{mfp}$ ). The variation of exposure buildup factors for all the selected ceramics with incident photon energy, penetration depth, and chemical composition has been studied.
\end{abstract}

\section{Introduction}

The recent nuclear reactor explosion in Japan emphasized the dire need of systematic and precise studies of dosimetric parameters of different type of materials. In the nuclear reactor, multienergetic photons were released, and for protection from these highly penetrating radiations, thick walls of concrete were built around the nuclear reactor. However, in case of nuclear accident, these highly penetrating radiations can travel longer distances and can cause harm to living organisms. In such a situation, the extent to which building materials can provide shielding from these harmful radiations is of utmost concern. Keeping this in mind, an attempt has been made to visualize the interaction of photons with one of the building material, namely, ceramics.

Ceramics are the composite materials in which the mechanical properties such as strength, modulus, toughness, wear resistance, and hardness are of primary interest. Despite possessing the strength and modulus values which are equal to or better than metals, these materials have chemical inertness and brittle fracture behavior. Considering such properties, ceramics have been selected to visualize the feasibility of using these materials as gamma ray shielding material.

The intensity of a gamma rays beam follows LambertBeer law $\left(I=I_{o} e^{-\mu x}\right)$ under three conditions which are (i) monochromatic radioactive source, (ii) thin absorbing material, and (iii) narrow beam geometry that should be used. In case, any of the three conditions has been violated, this law no longer holds. However, violation of the law can be maintained using the correction factor $B$, which is known as buildup factor. Different researchers have conducted experimental and theoretical studies in different type of materials. Several methods (geometric progression (G.P.) fitting method [1-3] and invariant embedding method [4]) have been used for the computation of buildup factors for different materials in different geometrical situations.

American Nuclear Society [2] provided a comprehensive set of standard data for exposure buildup factor which includes twenty three elements, two compounds, and one mixture in the energy range of 0.015 to $15.0 \mathrm{MeV}$ and up to the penetration depth of $40 \mathrm{mfp}$.

In our previous works $[5,6]$, the various types of buildup factors and different methods/codes available to compute 
the buildup factor have been already discussed. Recently, different researchers had contributed in providing gamma ray buildup factor data for different materials such as for thermoluminescent dosimetric materials [7], flyash concretes [8], human tissue [9], teeth [10, 11], some essential amino acids, fatty acids, and carbohydrates [12], and samples from the earth, moon, and mars [13].

In the present work, G.P. fitting method has been adopted to compute exposure buildup factors at some incident photon energies in the range of 0.015 to $15 \mathrm{MeV}$ with penetration depth up to $40 \mathrm{mfp}$ for some ceramics.

\section{Computational Work}

The computational work of exposure buildup factor for the selected ceramics has been divided into three parts. The first part deals with the computation of equivalent atomic number $\left(Z_{\text {eq }}\right)$ for the selected ceramics in the energy region of $15.0 \mathrm{keV}$ to $15.0 \mathrm{MeV}$. The second part concerns with the computation of G.P. fitting parameters, and finally in the third part, exposure buildup factor values have been computed in the same energy region.

2.1. Computations of Equivalent Atomic Numbers $\left(Z_{e q}\right)$. For the computation of $Z_{\text {eq }}$, the values of Compton partial attenuation coefficient $\left(\mu_{\mathrm{Comp}}\right)$ and the total attenuation coefficients ( $\mu_{\text {total }}$ ) were obtained in $\mathrm{cm}^{2} / \mathrm{g}$ for the selected ceramics in the energy range of 0.015 to $15.0 \mathrm{MeV}$ using WinXCom program [14]. The values of $Z_{\text {eq }}$ for the selected ceramics were computed by matching the ratio $R\left(\mu_{\text {Comp }} / \mu_{\text {total }}\right)$ of a particular ceramics at a selected energy with the corresponding ratio of an element at the same energy. In case the value of ratio lies between two ratios for known successive elements, the value $Z_{\text {eq }}$ was then interpolated using the following logarithmic interpolation formula [6]:

$$
Z_{\mathrm{eq}}=\frac{Z_{1}\left(\log R_{2}-\log R\right)+Z_{2}\left(\log R-\log R_{1}\right)}{\left(\log R_{2}-\log R_{1}\right)},
$$

where $Z_{1}$ and $Z_{2}$ are the atomic numbers of elements corresponding to the $\left(\mu_{\text {Comp }} / \mu_{\text {total }}\right)$ ratios, $R_{1}$ and $R_{2}$, respectively, and $R\left(\mu_{\text {Comp }} / \mu_{\text {total }}\right)$ is the ratio for the selected ceramic at a particular energy, which lies between ratios $R_{1}$ and $R_{2}$.

2.2. Computations of G.P. Fitting Parameters. American National Standards [2] provided the exposure G.P. fitting parameters of 23 elements $\left({ }_{4} \mathrm{Be}-{ }_{8} \mathrm{O},{ }_{11} \mathrm{Na}_{16} \mathrm{~S},{ }_{18} \mathrm{Ar}-{ }_{20} \mathrm{Ca},{ }_{26} \mathrm{Fe}\right.$, ${ }_{29} \mathrm{Cu}, \mathrm{Mo}, \mathrm{Sn}, \mathrm{La}, \mathrm{Gd}, \mathrm{W},{ }_{82} \mathrm{~Pb}$, and $\left.{ }_{92} \mathrm{U}\right)$, one compound (water), and two mixtures (air and concrete) in the energy range of 0.015 to $15.0 \mathrm{MeV}$ and up to a penetration depth of $40 \mathrm{mfp}$. The computed values of $Z_{\mathrm{eq}}$ for the selected ceramics were used to interpolate G.P. fitting parameters ( $b, c, a, X_{k}$, and $d$ ) for the exposure buildup factor using the following logarithmic interpolation formula [6]:

$$
P=\frac{P_{1}\left(\log Z_{2}-\log Z_{\mathrm{eq}}\right)+P_{2}\left(\log Z_{\mathrm{eq}}-\log Z_{1}\right)}{\log Z_{2}-\log Z_{1}},
$$

where $Z_{1}$ and $Z_{2}$ are the elemental atomic numbers between which the equivalent atomic number $Z_{\mathrm{eq}}$ of the chosen ceramic lies. $P_{1}$ and $P_{2}$ are the values of G.P. fitting parameters corresponding to the atomic numbers $Z_{1}$ and $Z_{2}$, respectively, at a given energy. Using the interpolation formula, G.P. fitting parameters for exposure buildup factors were computed at the selected incident photon energies for the chosen ceramics.

2.3. Computations of Buildup Factors. The computed G.P. fitting parameters $\left(b, c, a, X_{k}\right.$, and $\left.d\right)$ were used to compute the exposure buildup factors for the selected ceramics in incident photon energy range of 0.015 to $15.0 \mathrm{MeV}$ and up to the penetration depth of $40 \mathrm{mfp}$ using following equations [2-4]:

$$
\begin{gathered}
B(E, x)=1+\frac{b-1}{K-1}\left(K^{x}-1\right), \quad \text { for } K \neq 1, \\
B(E, x)=1+(b-1) x, \quad \text { for } K=1,
\end{gathered}
$$

where

$$
\begin{array}{r}
K(E, x)=c x^{a}+d \frac{\tanh \left(x / X_{k}-2\right)-\tanh (-2)}{1-\tanh (-2)}, \\
\text { for } x \leqslant 40 \mathrm{mfp}
\end{array}
$$

\section{Results and Discussion}

The variation of exposure buildup factor with the incident photon energy in the range of 0.015 to $15.0 \mathrm{MeV}$ has been shown in Figure 1 for BN at some of the penetration depths $(1,5,10,20,30$, and $40 \mathrm{mfp})$. For the fixed penetration depth of $1 \mathrm{mfp}$, at lower incident photon energy $(0.015 \mathrm{MeV})$, the value of energy absorption buildup factor is small, and it increases with the increase in incident photon energy, reaches a maximum value in the intermediate energy region, and after that starts decreasing with the further increase in the incident photon energy.

In lower and higher energy regions photo-electric and pair productions are most dominant processes (in which complete absorption of photon takes place), which result in minimum value of buildup factor. While in the intermediate energy region, Compton scattering is the dominant photon interaction process, which results only in the energy degradation of the photon and not the complete absorption. Hence the photons will pile up and give rise to peak. Similar trend has been observed at higher penetration depths of the ceramic.

The dominant range of Compton scattering process can be expressed in the range of $E_{\text {photo-Comp }}$ and $E_{\text {Comp-pair prod }}$ where $E_{\text {photo-Comp }}$ represents the energy for which both photoelectric absorption and Compton scattering show almost equal value for mass attenuation coefficient. Whereas $E_{\text {Comp-pair prod }}$ represents the energy for which both Compton scattering and pair production processes show equal dominance. For boron nitride (least $Z_{\text {eq }}$ ceramic), the value of $E_{\text {photo-Comp }}$ is about $23 \mathrm{keV}$ (the corresponding value of mass attenuation coefficient $\left(\mu_{m}\right)$ at which energy is $0.160 \mathrm{~cm}^{2} / \mathrm{g}$ ) 


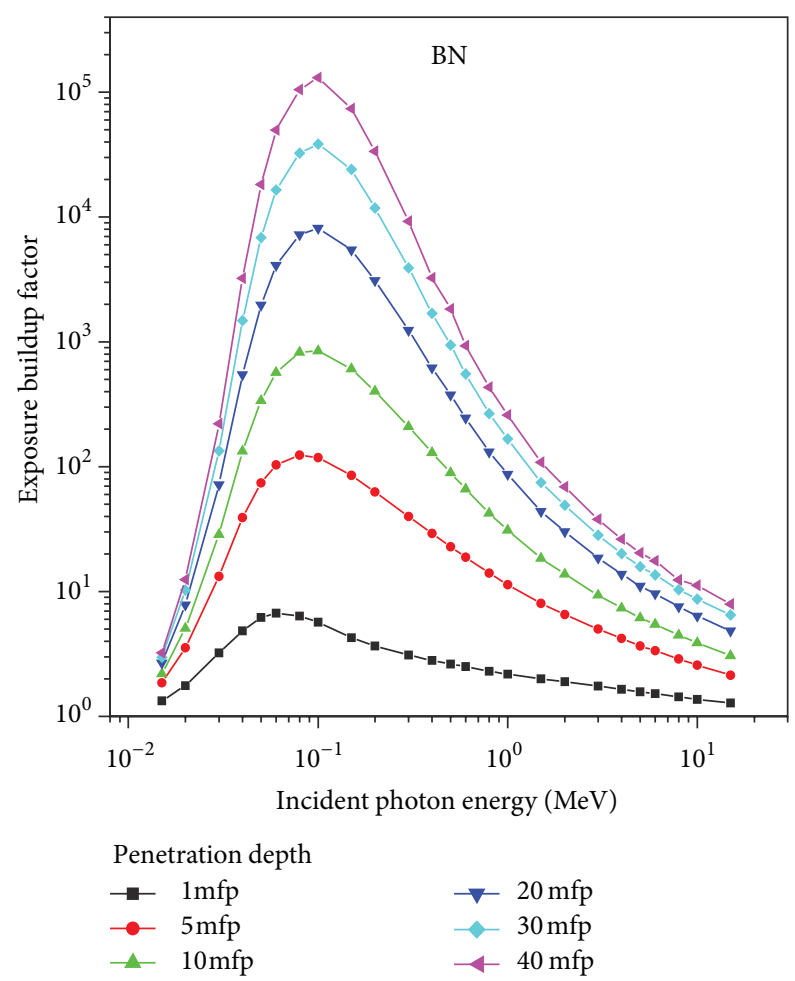

FIGURE 1: Variation of exposure buildup factor with incident photon energy for BN.

and the value of $E_{\text {Comp-pair prod }}$ is $28 \mathrm{MeV}$ (the corresponding value of mass attenuation coefficient $\left(\mu_{m}\right)$ at which energy is $0.68 \times 10^{-2} \mathrm{~cm}^{2} / \mathrm{g}$ ). Whereas for ferrite (highest $Z_{\text {eq }}$ ceramic), the value of $E_{\text {photo-Comp }}$ is about $100 \mathrm{keV}$ (the corresponding value of mass attenuation coefficient $\left(\mu_{m}\right)$ at which energy is $0.14 \mathrm{~cm}^{2} / \mathrm{g}$ ), and the value of $E_{\mathrm{Comp} \text {-pair prod }}$ is $12 \mathrm{MeV}$ (the corresponding value of mass attenuation coefficient $\left(\mu_{m}\right)$ at which energy is $\left.0.13 \times 10^{-1} \mathrm{~cm}^{2} / \mathrm{g}\right)$. Since, the exposure buildup factor is the result of multiple Compton scattering processes, hence the study of buildup factor is of utmost importance between the values of $E_{\text {photo-Comp }}$ and $E_{\text {Comp-pair prod. Similarly, for other ceramics like magnesium }}$ diboride, silicon carbide, titanium carbide, and ferrite, the dominant range for Compton scattering process lies in between $15.0 \mathrm{keV}$ and $15.0 \mathrm{MeV}$.

Further, it has been also observed that the ceramics with low $Z_{\text {eq }}$ show large Compton scattering dominant range (as in case of $\mathrm{BN}$ ), whereas for ceramics with comparatively high $Z_{\text {eq }}$, Compton scattering dominance region is less (as in cases of ferrite and titanium carbide). Exposure buildup factor as a function of penetration depth up to 40 mean free path for the selected ceramics has been shown in Figure 2 for $\mathrm{BN}$ at some of the selected incident photon energies $(0.015,0.10$, $0.50,5.00$, and $15.0 \mathrm{MeV})$. It has been observed that at all the selected energies, exposure buildup factor increases with the increase in penetration depth of $\mathrm{BN}$. It may be due to the reason that as thickness of ceramic increases, the probability of multiple Compton scatterings also increases, and hence the exposure buildup factor increases. Similar trend has been observed for other ceramics.

However, the increasing rate was found to be slow for lower and higher incident photon energies, and rapid increase was observed in case of intermediate energy region. The slower increasing rate in the lower and higher energy regions was due to the dominance of different photon absorption processes in these energy regions (photoelectric effect in the lower energy region and pair production in the higher energy region) which results in the complete absorption of gamma photons in the interacting medium, whereas in the intermediate energy region the dominant process is the Compton scattering, which results only in the energy degradation of photons. Hence, there is a finite possibility of the photon to reach the detector even for the large penetration depths of the ceramics, and hence maximum violation of Lambert-Beer equation has been observed.

Further, the increasing rate of exposure buildup factor with the penetration depth is more rapid up to the certain incident photon energy $(0.1 \mathrm{MeV})$, where the Compton scattering process is most dominant process, and after this the increasing rate of exposure buildup factor becomes slower for higher energies.

All the selected ceramics have different chemical composition and hence different equivalent atomic number $\left(Z_{\mathrm{eq}}\right)$. So, to study the chemical composition dependence of different ceramics on exposure buildup factor, exposure buildup factor for all the selected ceramics has been plotted against the incident photon energy at fixed penetration depths of $1,5,10$, and $40 \mathrm{mfp}$ and has been shown in Figures 3, 4, 5, and 6. From these figures, it has been observed that for all the selected ceramics, exposure buildup factor values are small at lower incident photon energies as well as higher incident photon energies and show maximum values in the intermediate energy region. It may be due to the same reason of dominance of different partial photon interaction processes in different energy regions. Among the selected ceramics, ferrite (highest $Z_{\text {eq }}$ ) shows the minimum value for the exposure buildup factor, whereas maximum values are observed for boron nitride (lowest $Z_{\text {eq }}$ ). It may be due to the reason that ferrite, which is a ceramic of oxygen $(Z=8$, weight fraction $=0.30)$ and iron $(Z=26$, weight fraction $=0.70)$, has the maximum equivalent atomic number due to the major contribution of iron. Whereas boron nitride consists of boron $(Z=5$, weight fraction $=0.44)$ and nitrogen $(Z=7$, weight fraction $=0.56)$ and has the minimum equivalent atomic number. From this observation, it can be concluded that exposure buildup factor is inversely proportional to the equivalent atomic number of the ceramics at lower penetration depths (below $10 \mathrm{mfp}$ ).

In Figure 5, for the fixed penetration depth of $10 \mathrm{mfp}$ of all the selected ceramics and for incident photon energy above $3 \mathrm{MeV}$, different ceramics show almost same values for exposure buildup factor. It signifies that, above certain incident photon energy (about $3 \mathrm{MeV}$ ), exposure buildup factor becomes almost independent of the chemical composition of the interacting material. Further, the selected ceramics mostly follow different crystal structures such as $\mathrm{BN}, \mathrm{SiC}$, and $\mathrm{MgB}_{2}$ follow hexagonal, TiC follows cubic, and $\mathrm{Fe}_{2} \mathrm{O}_{3}$ follows rhombohedral structure. Since different 


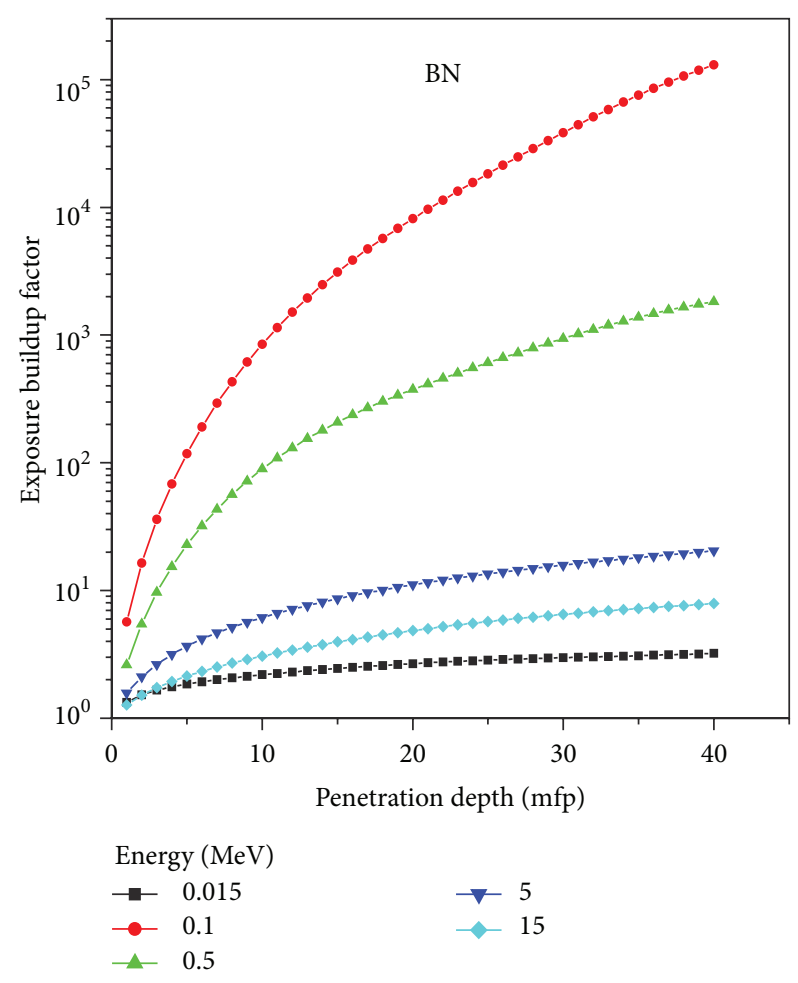

FIGURE 2: Variation of exposure buildup factor with penetration depth of BN.

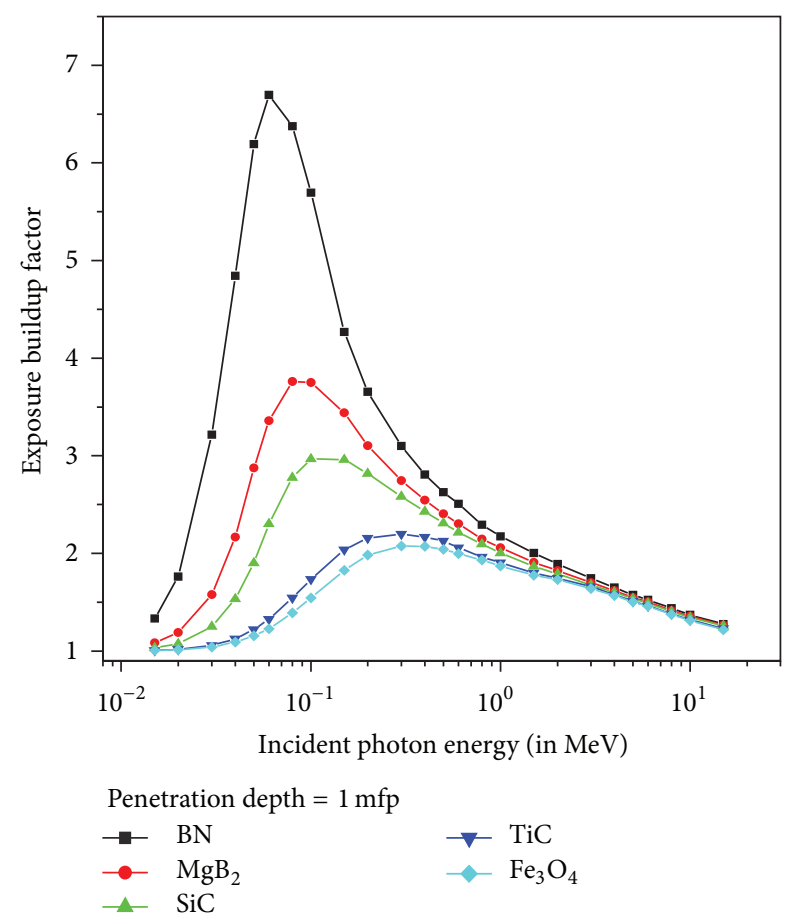

FIGURE 3: Variation of exposure buildup factor with incident photon energy for all ceramics at $1 \mathrm{mfp}$.

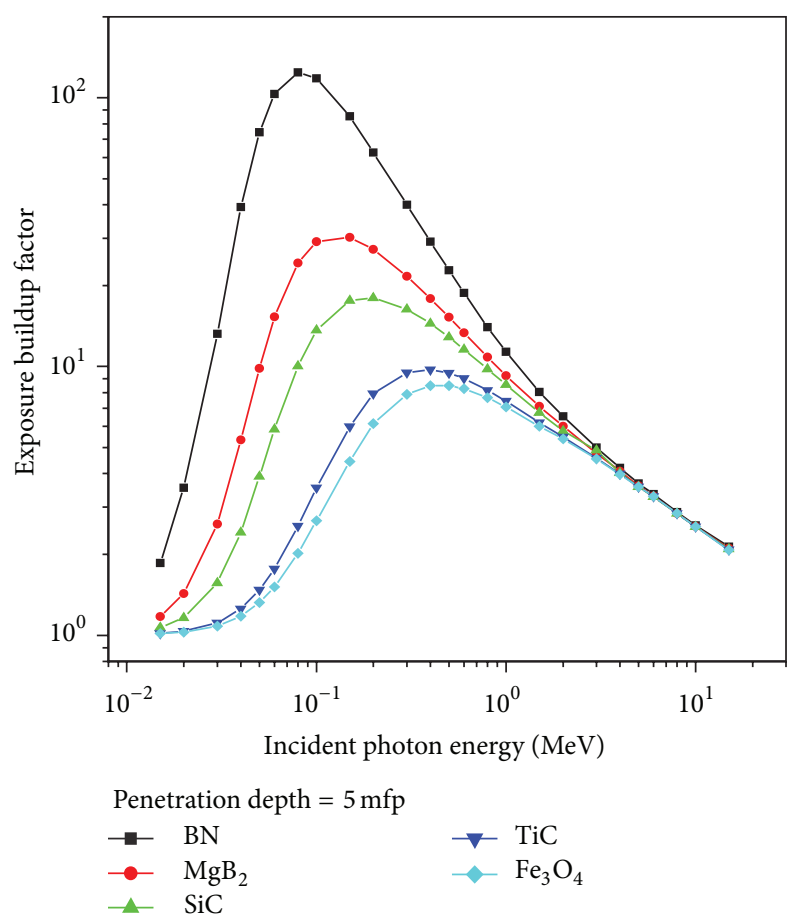

FIGURE 4: Variation of exposure buildup factor with incident photon energy for all ceramics at $5 \mathrm{mfp}$.

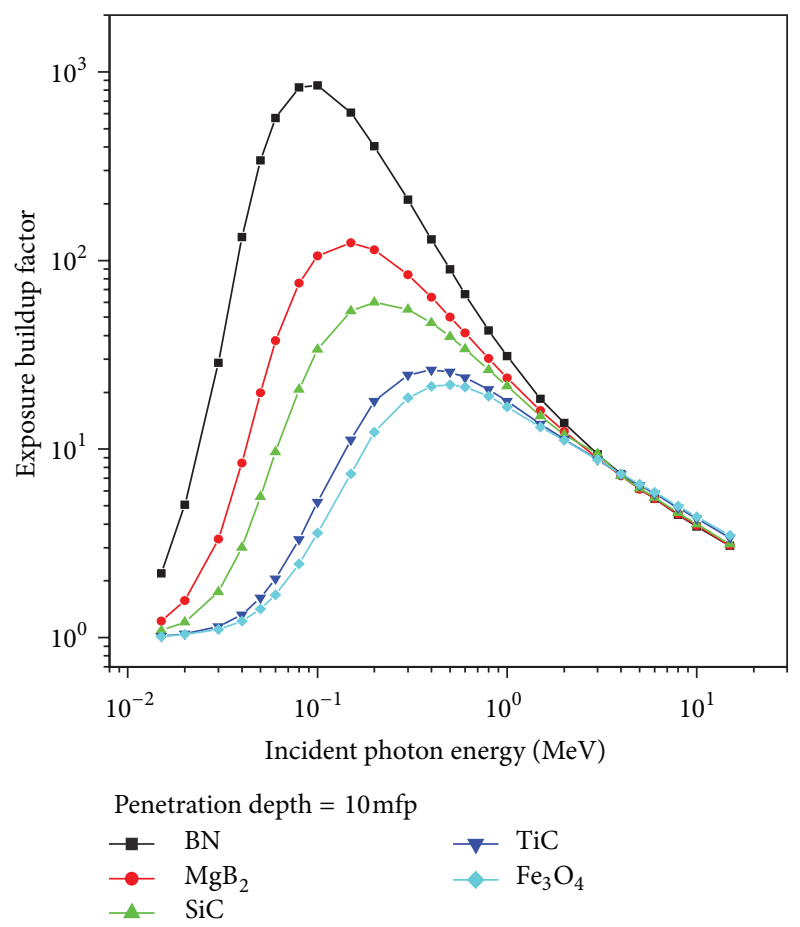

FIGURE 5: Variation of exposure buildup factor with incident photon energy for all ceramics at $10 \mathrm{mfp}$. 


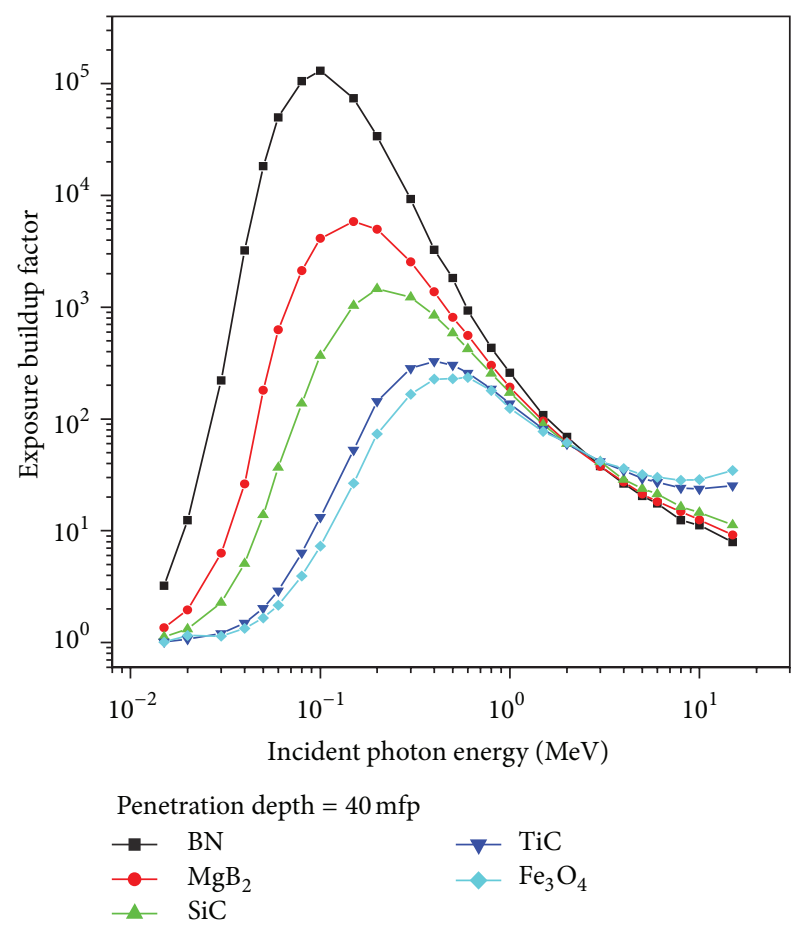

FIGURE 6: Variation of exposure buildup factor with incident photon energy for all ceramics at $40 \mathrm{mfp}$.

ceramics follow different crystal structure, the same values for exposure buildup factor above $3 \mathrm{MeV}$ photon energy and at the penetration depth of $10 \mathrm{mfp}$ suggest that exposure buildup factor becomes independent of crystal structure.

However, in Figure 6, which shows the variation of exposure buildup factor for all the selected ceramics at the fixed penetration depths of $40 \mathrm{mfp}$, reversal in the trend of exposure buildup factor values has been observed above $3 \mathrm{MeV}$. That is above the incident photon energy of $3 \mathrm{MeV}$, exposure buildup factor shows maximum values for ferrite (highest $Z_{\text {eq }}$ ceramic) and minimum values for boron nitride (lowest $Z_{\text {eq }}$ ceramic); that is, the exposure buildup factor becomes directly proportional to the equivalent atomic number of the ceramic. It may be due to the reason that pair production initiates from $1.022 \mathrm{MeV}$, and its dominance increases with the increase in photon energy, and it results in the formation of an electron and a positron. For smaller penetration depths (below 10 mean free path) of the ceramics, these particles escape either from the material or after multiple collision within the ceramic comes to rest and further annihilates, that is, creates two secondary gamma rays of $0.511 \mathrm{MeV}$, which escapes from the ceramic material. With the increase in penetration depth (above $10 \mathrm{mfp}$ ), these secondary gamma rays (due to annihilation) contribute in increasing the intensity of primary gamma rays and try to compensate for the decrease in primary gamma rays due to pair production. With the further increase in the penetration depth, that is, for larger penetration depths, the probability of creation of secondary gamma rays increases, and hence the contribution of these secondary gamma rays towards exposure buildup factor also increases.

\section{Conclusions}

From the present studies, the following conclusions can be drawn.

(i) Exposure buildup factor increases with the increase in penetration depth (40 mfp).

(ii) Exposure buildup factor shows the following different trends with incident photon energy.

(a) For the entire energy region $(0.015-15.0 \mathrm{MeV})$, in case of small penetration depths (below $10 \mathrm{mfp}$ ), exposure buildup factor is inversely proportional to the $Z_{\mathrm{eq}}$.

(b) In the higher energy region (above $3 \mathrm{MeV}$ for the selected ceramics), there exists a penetration depth (about $10 \mathrm{mfp}$ in the present case), for which exposure buildup factor becomes almost independent of the $Z_{\mathrm{eq}}$ or the chemical composition of the ceramics.

(c) In the higher energy region (above $3 \mathrm{MeV}$ ), for large penetration depths (above $15 \mathrm{mfp}$ ), exposure buildup factor becomes directly proportional to $Z_{\mathrm{eq}}$.

Among the selected ceramics, ferrite $\left(\mathrm{Fe}_{3} \mathrm{O}_{4}\right)$ offers better gamma ray shielding.

\section{References}

[1] Y. Sakamoto, S. Tanaka, and Y. Harima, "Interpolation of gamma ray build-up factors for point isotropic source with respect to atomic number," Nuclear Science and Engineering, vol. 100, pp. 33-42, 1988.

[2] American National Standard Institute, "Gamma-ray attenuation coefficients and buildup factors for engineering materials," Report ANSI/ANS 6.4.3, 1991.

[3] Y. Harima, Y. Sakamoto, S. Tanaka, and M. Kawai, "Validity of the geometrical progression formula in approximating gammaray buildup factors," Nuclear Science and Engineering, vol. 94, pp. 24-35, 1986.

[4] A. Shimizu and H. Hirayama, "Calculation of gamma-ray buildup factors up to depths of $100 \mathrm{mfp}$ by the method of invariant embedding," Nuclear Science and Technology, vol. 40, pp. 192-200, 2003.

[5] P. S. Singh, T. Singh, and P. Kaur, "Variation of energy absorption buildup factors with incident photon energy and penetration depth for some commonly used solvents," Annals of Nuclear Energy, vol. 35, pp. 1093-1097, 2008.

[6] T. Singh, N. Kumar, and P. S. Singh, "Chemical composition dependence of exposure buildup factors for some polymers," Annals of Nuclear Energy, vol. 36, no. 1, pp. 114-120, 2009.

[7] S. R. Manohara, S. M. Hanagodimatha, and L. Gerwardb, "Energy absorption buildup factors for thermoluminescent dosimetric materials and their tissue equivalence," Radiation Physics and Chemistry, vol. 79, no. 5, pp. 575-582, 2010. 
[8] S. Singh, S.S. Ghumman, C. Singh, K. S. Thind, and G. S. Mudahar, "Buildup of gamma ray photons in flyash concretes: a study," Annals of Nuclear Energy, vol. 37, pp. 681-684, 2010.

[9] M. Kurudirek, B. Doğan, M. İngeç, N. Ekinci, and Y. Özdemir, "Gamma-ray energy absorption and exposure buildup factor studies in some human tissues with endometriosis," Applied Radiation and Isotopes, vol. 69, pp. 381-388, 2011.

[10] H. C. Manjunatha and B. Rudraswamy, "Computation of exposure build-up factors in teeth," Radiation Physics and Chemistry, vol. 80, no. 1, pp. 14-21, 2011.

[11] M. Kurudirek and S. Topcuoglu, "Investigation of human teeth with respect to the photon interaction, energy absorption and buildup factor," Nuclear Instruments and Methods in Physics Research B, vol. 269, no. 10, pp. 1071-1081, 2011.

[12] M. Kurudirek and Y. Ozdemir, "A comprehensive study on energy absorption and exposure buildup factors for some essential amino acids, fatty acids and carbohydrates in the energy range $0.015-15 \mathrm{MeV}$ up to 40 mean free path," Nuclear Instruments and Methods in Physics Research B, vol. 269, no. 1, pp. 7-19, 2011.

[13] M. Kurudirek, B. Dogan, Y. Özdemir, A. Camargo Moreira, and C. R. Appoloni, "Analysis of some Earth, Moon and Mars samples in terms of gamma ray energy absorption buildup factors: Penetration depth, weight fraction of constituent elements and photon energy dependence," Radiation Physics and Chemistry, vol. 80, pp. 354-364, 2011.

[14] L. Gerward, N. Guilbert, K. Bjørn Jensen, and H. Levring, "X-ray absorption in matter. Reengineering XCOM," Radiation Physics and Chemistry, vol. 60, no. 1-2, pp. 23-24, 2001. 

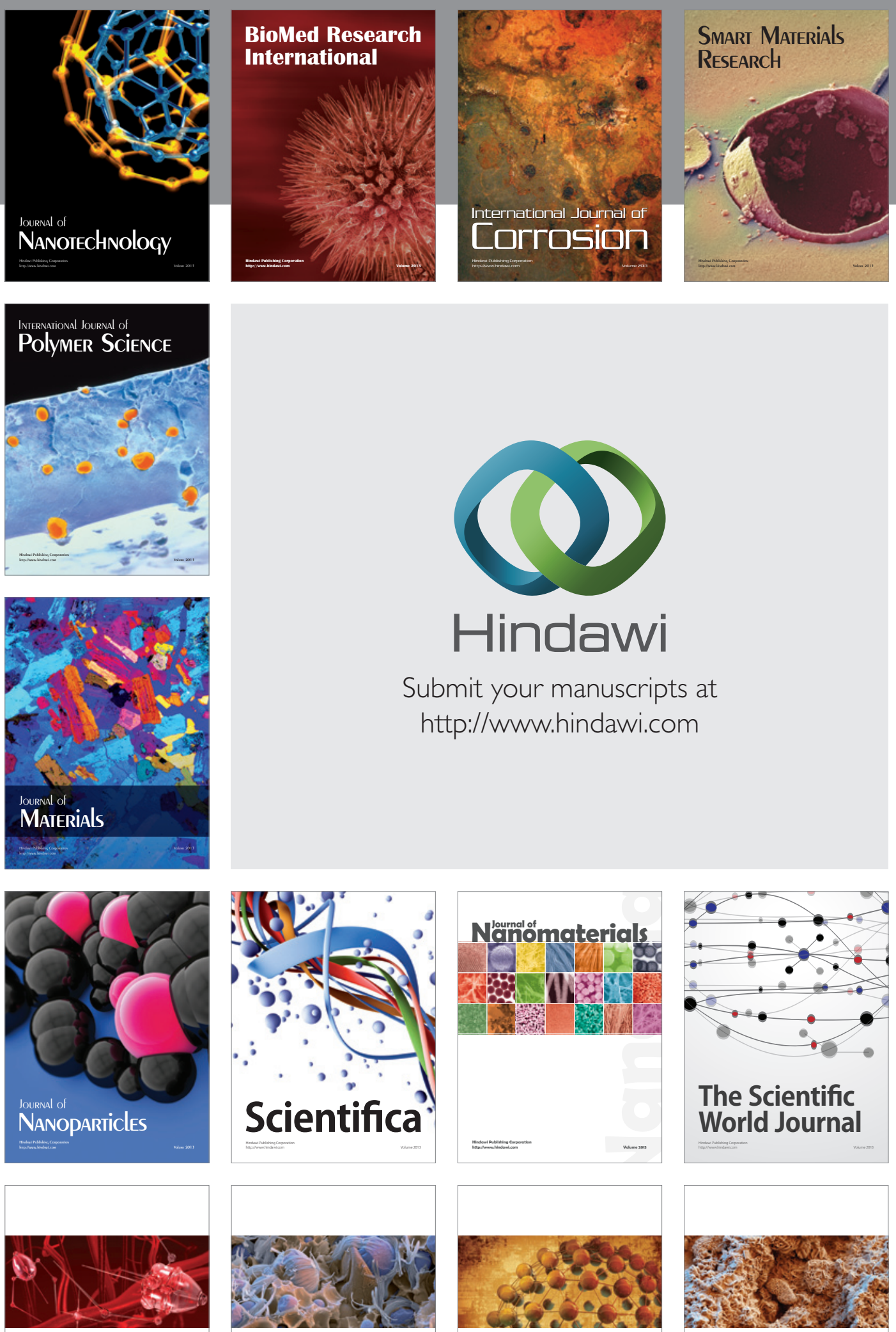

ISRN

Nanotechnology
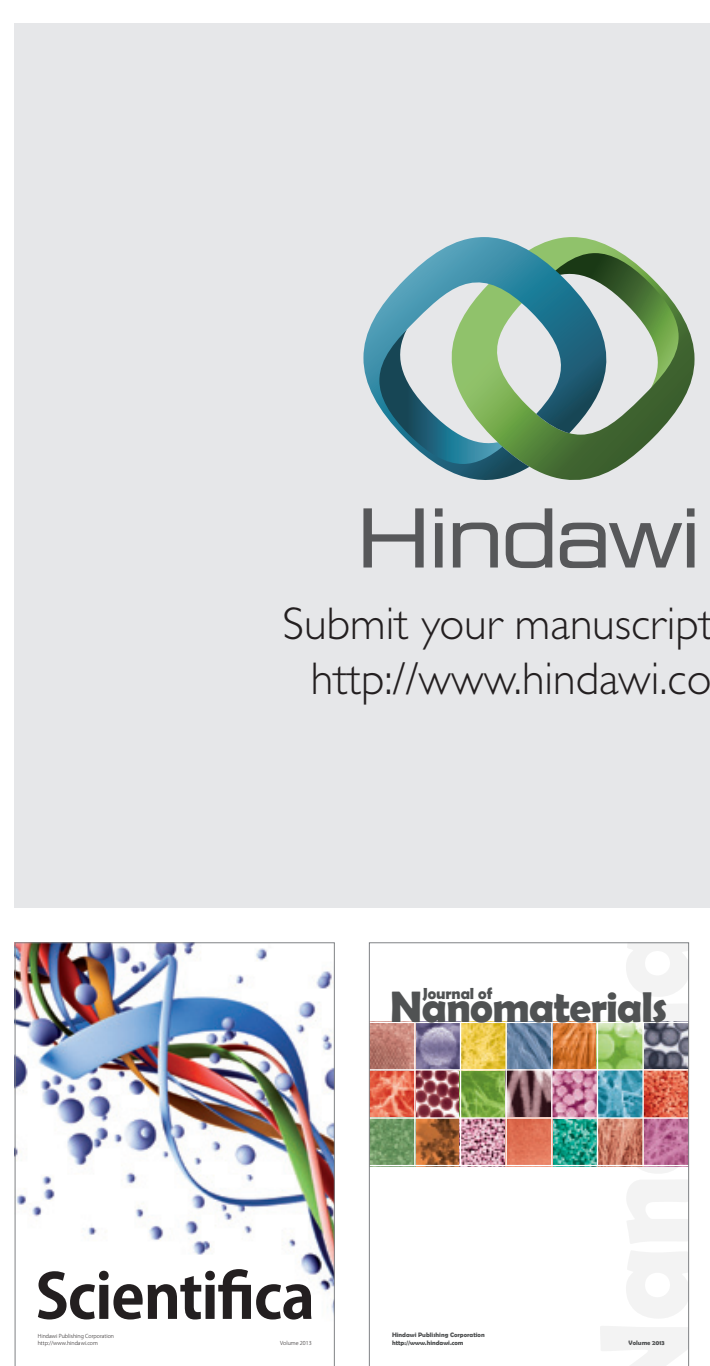

Submit your manuscripts at http://www.hindawi.com
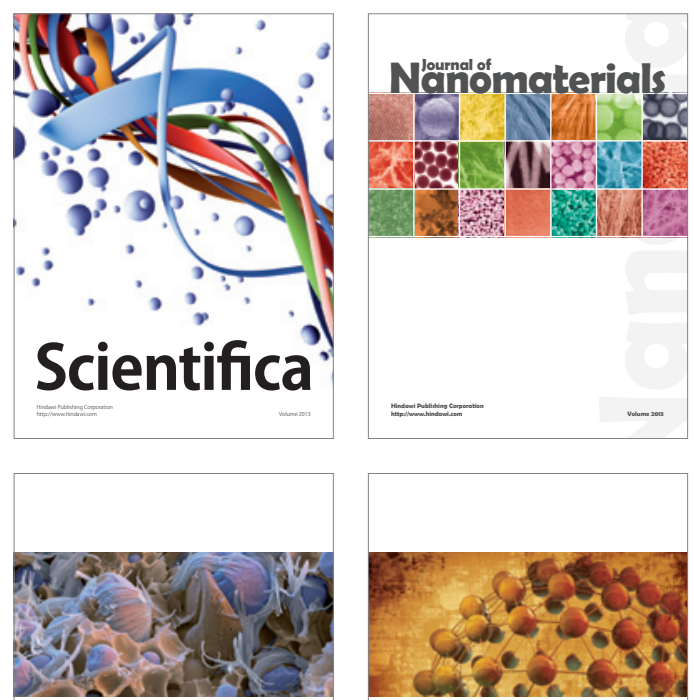

ISRN

Polymer Science

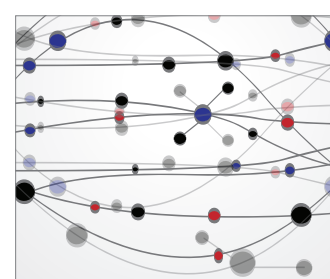

The Scientific World Journal

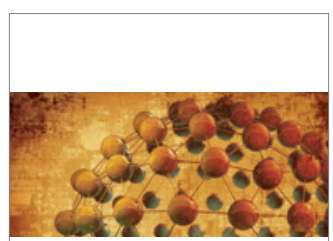

ISRN

Materials Science

\section{World Jounal}

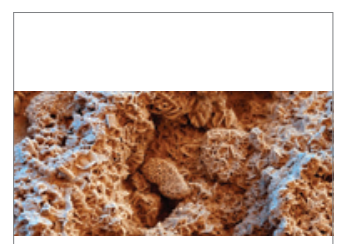

ISRN

Corrosion
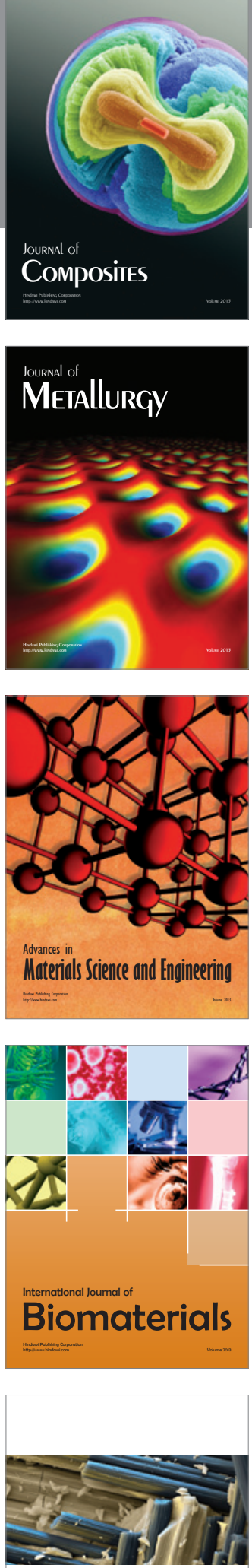

ISRN

Ceramics 